\title{
A Novel SCN5A Variant Causes Temperature-Sensitive Loss Of Function in a Family with Symptomatic Brugada Syndrome, Cardiac Conduction Disease, and Sick Sinus Syndrome
}

\author{
Karolina Sanner $^{\mathrm{a}} \quad$ Johanna Mueller-Leisse $^{\mathrm{b}}$ Christos Zormpas $^{\mathrm{b}}$ David Duncker $^{\mathrm{b}}$ \\ Andreas Leffler ${ }^{a}$ Christian Veltmann ${ }^{b}$ \\ aDepartment of Anesthesiology and Intensive Care Medicine, Hannover Medical School, Hannover, Germany; \\ ${ }^{b}$ Rhythmology and Electrophysiology, Department of Cardiology and Angiology, Hannover Medical School, Hannover, \\ Germany
}

\section{Keywords}

Nav1.5 · Brugada syndrome · Channelopathy · Cardiac conduction disease

\begin{abstract}
Introduction: Brugada syndrome (BrS) is an inherited arrhythmia syndrome associated with an increased risk of sudden cardiac death. SCN5A is the most important diseasemodifying gene for BrS, but many SCN5A variants have not been functionally characterized. Furthermore, the temperature dependency of SCN5A is only rarely explored in in vitro analyses. Methods: The clinical phenotype of the affected family was assessed by medical history, ECGs and ajmaline challenge. Whole-cell patch clamp recordings were performed on HEK 293T cells expressing Nav1.5-G1712S, a novel SCN5A variant found in the symptomatic family. Results: Three male family members had experienced sudden cardiac death, sudden cardiac arrest, and rhythmogenic syncopes. Beside a positive ajmaline challenge with demarcation of a Brugada type 1 ECG, 1 patient also showed evidence of symptomatic cardiac conduction disease and sick sinus syndrome (SSS). In patch clamp analyses, Nav1.5-G1712S generated reduced peak currents as compared to the wild type. At body temperature, Nav1.5-G1712S additionally exhibited an
\end{abstract}

karger@karger.com www.karger.com/crd

Karger $\frac{1}{\%}$
C 2021 The Author(s).

Published by S. Karger AG, Basel

This article is licensed under the Creative Commons Attribution 4.0 International License (CC BY) (http://www.karger.com/Services/ OpenAccessLicense). Usage, derivative works and distribution are permitted provided that proper credit is given to the author and the original publisher. enhanced slow inactivation and an impaired recovery from inactivation. Conclusion: We conclude that G1712S is a pathogenic SCN5A loss-of function mutation at physiological temperature associated with an overlapping presentation of BrS, SSS, and cardiac conduction disease.

(c) 2021 The Author(s).

Published by S. Karger AG, Basel

\section{Introduction}

Brugada syndrome $(\mathrm{BrS})$ is an inherited arrhythmia syndrome associated with an increased risk of malignant cardiac arrhythmias. The variable phenotype of $\mathrm{BrS}$ makes an accurate individual risk stratification prior to the implementation of invasive preventive treatment options like an implantable cardioverter-defibrillator (ICD) or a pacemaker challenging [1]. Overlapping phenotypes with other inherited arrhythmia syndromes such as sick sinus syndrome (SSS), cardiac conduction disease, and long QT syndrome complicate the management of these patients [1,2]. Genetic testing is being increasingly performed in symptomatic patients and their family members. A thorough under-

K.S. and J. M.-L. contributed equally to the article.

Correspondence to:

Christian Veltmann, veltmann.christian@mh-hannover.de 
standing of the pathogenicity of any given variant may lead to a more efficient risk stratification [1]. SCN5A encodes for the $\alpha$-subunit of the cardiac sodium channel Nav1.5 and is probably the most relevant diseasemodifying gene for BrS [3]. Variants of SCN5A are identified in about $20 \%$ of patients, but only some of them are actually considered causative $[4,5]$. Typically, these variants associated with BrS display loss-of-function properties as determined by an in vitro patch clamp $[1,6]$. These experiments are usually conducted at room temperature, which may be a critical limitation as some biophysical properties of sodium channels are temperature-sensitive [7-9]. We identified the SCN5A variant G1712S in a family with symptomatic BrS. This variant was already identified in a single patient with BrS [6], but it has not been characterized by means of in vitro electrophysiology, and no clinical data have been published. In this study, we present the phenotype of the affected family and biophysical properties of the mutant Nav1.5-G1712S.

\section{Materials and Methods}

\section{Clinical Analysis}

A family with BrS and familial sudden cardiac death was identified. All family members were encouraged to undergo complete clinical and genetic workup including medical and family history, physical examination, repetitive baseline ECGs, stress test, and ajmaline challenge. The study was approved by the Ethics Committee of Hannover Medical School (No. 1673-2013).

\section{Mutagenesis}

Human (h) Nav1.5 cDNA was PCR amplified and subcloned into the pTracer-SV40 vector (Invitrogen, Carlsbad, CA, USA). Nav1.5-G1712S and Nav1.5-G1712C mutants were generated by site-directed mutagenesis of hNav1.5 cDNA in pTracer-SV40 using the QuikChange Lightning Site-Directed Mutagenesis Kit (Agilent, Waldbronn, Germany) according to the instructions of the manufacturer. Mutants were sequenced to verify intended amino acid exchanges and to exclude further channel mutations.

\section{Cell Culture and Heterologous Expression}

All experiments were performed in accordance with the requirements of the local authorities (Gewerbeaufsicht, Niedersachsen, Germany). HEK 293T cells were cultured in standard Dulbecco's modified Eagle medium (Gibco Life Technologies, Karlsruhe, Germany) supplemented with $10 \%$ fetal bovine serum (Biochrom, Berlin, Germany), $100 \mathrm{U} / \mathrm{mL}$ penicillin and $100 \mu \mathrm{g} / \mathrm{mL}$ streptomycin (Gibco Life Technologies, Karlsruhe, Germany) in a $37^{\circ} \mathrm{C}$ incubator in air with $5 \% \mathrm{CO}_{2}$. Wild-type hNav1.5, Nav1.5G1712S, and Nav1.5-G1712C were transfected with JetPEI DNA Tranfection Reagent (Polyplus-transfection, New York, NY, USA) using $3 \mu \mathrm{g}$ of cDNA and $6 \mu \mathrm{g}$ of JetPEI reagent. Patch Clamp recordings were performed within $72 \mathrm{~h}$.

Nav1.5-G1712S Is a Variant of SCN5A

Associated with Brugada Syndrome

\section{Patch Clamp Experiments}

$\mathrm{Na}^{+}$currents from transfected HEK 293T cells were recorded using the whole-cell configuration of the patch clamp technique with an HEKA EPC9 patch-clamp amplifier (HEKA Elektronik, Lambrecht, Germany). Data were sampled at $20 \mathrm{kHz}$ and filtered at $5 \mathrm{kHz}$. Data were recorded using Pulse software (v 8.80; HEKA Electronik) and analyzed with Fitmaster software (HEKA Electronik, Lambrecht, Germany). Curve fitting and statistical analyses were performed using Origin 8.5 software (Microcal Software, Northampton, MA, USA). The bath solution contained (mM) 70 $\mathrm{NaCl}, 70$ choline chloride, $3 \mathrm{KCl}, 1 \mathrm{CaCl}_{2}, 1 \mathrm{MgCl}_{2}, 10 \mathrm{HEPES}$, and 15 glucose. $\mathrm{pH}$ was adjusted to 7.4 with tetraethylammonium hydroxide. The pipette solution contained (mM) $140 \mathrm{CsF}, 10 \mathrm{NaCl}$, 10 HEPES, and 1 EGTA, pH 7.4 (CsOH). Pipettes were pulled from borosilicate glass (GB 100 TF-8P; Science Products, Berlin, Germany), and their resistance ranged from 1.0 to $2.5 \mathrm{MOhm}$. Serial resistance $(5.2 \pm 2.4 \mathrm{MOhm})$ was compensated by $60-80 \%$ to $\mathrm{min}-$ imize voltage errors. Capacitance artifacts were compensated using the amplifier circuitry. Patch clamp experiments were carried out at $22-24^{\circ} \mathrm{C}$ (room temperature) or at $35-37^{\circ} \mathrm{C}$. For the experiments at physiological temperature, the external solution was preheated to $37^{\circ} \mathrm{C}$, and during the experiments, the temperature was maintained using a heating plate filled with temperature-controlled solution.

\section{Statistical Analysis}

Clinical and electrophysiological data were presented as mean $\pm \mathrm{SD}$ and mean \pm SE, respectively. The $\chi^{2}$ test and Fisher's exact test were used for comparison of categorical variables. Statistical significance was calculated with one-way ANOVA with the post hoc Tukey test. A $p$ value $<0.05$ was considered statistically significant. Calculations were performed using Origin 8.5 (Microcal Software, Northhampton, MA, USA). Data are presented as mean \pm standard error of mean.

\section{Results}

\section{Family History}

The index patient (III-4, Fig. 1a, b) survived sudden cardiac arrest at the age of 11 months. The initial rhythm detected was ventricular fibrillation. The boy suffered severe brain damage after $1 \mathrm{~h}$ of cardiopulmonary resuscitation. The parents were asymptomatic, but the mother (II-2) had lost 2 pregnancies, and her father (I-1) had died of sudden cardiac arrest at the age of 38 years. The initial diagnostic workup of the boy (III-4) including echocardiography, repeat ECGs, and Holter ECG did not result in any diagnosis. Genetic testing revealed a heterozygous missense point mutation on SCN5A, leading to the exchange of a glycine $(G)$ to serine $(S)$ at position 1,712 (Gly1712Ser; 5134G $>$ A). The same SCN5A variant was detected in the mother. After considering the family history and according to her personal wish, the mother received an ICD for primary prophylaxis. Two years later, 


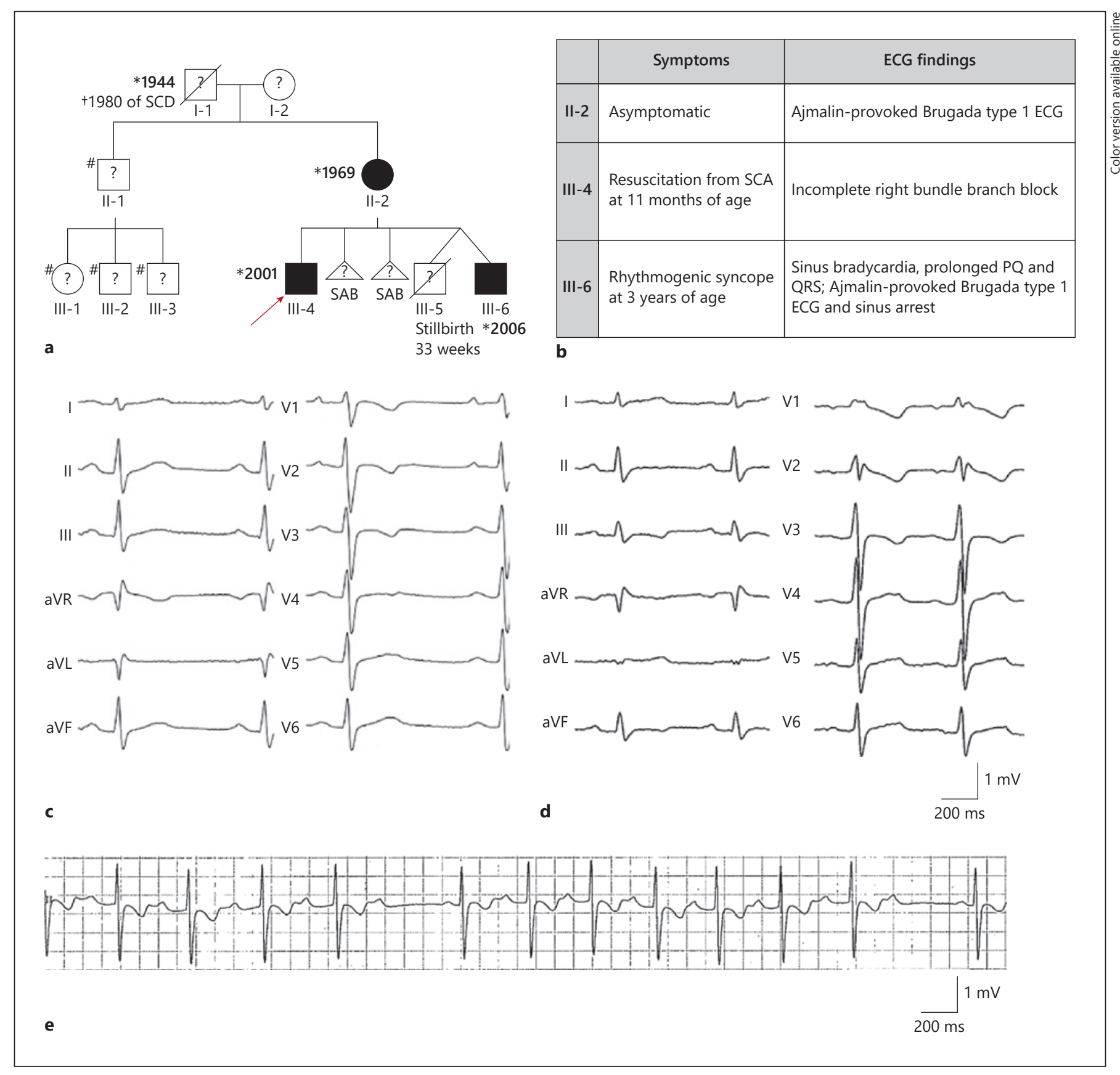

Fig. 1. a Family pedigree with the index patient indicated by the red arrow. $\mathbf{b}$ Table giving clinical parameters of affected patients. $\mathrm{SAB}$, spontaneous abortion; SCA, sudden cardiac arrest; \#, no clin-

she gave birth to male twins. One was a stillborn, and the other (III-6) was born healthy but tested positive for SCN5A-G1712S. At the age of 3 years, the surviving twin suffered from a suspected rhythmogenic syncope during a febrile episode and received an ICD/pacemaker for sec- ical data available. c, d Representative resting ECGs of III-6 (c, at the age of 7 years) and III- 4 (d, at the age of 3 years). e Intrahospital ECG monitoring of III-6 after syncope.

ondary prophylaxis. After the ICD implantation, both patients (II-2 and III-6) have not experienced any recurrences of spontaneous arrhythmia nor any device-associated complications. No relevant clinical information was available from II-1 and his children. 


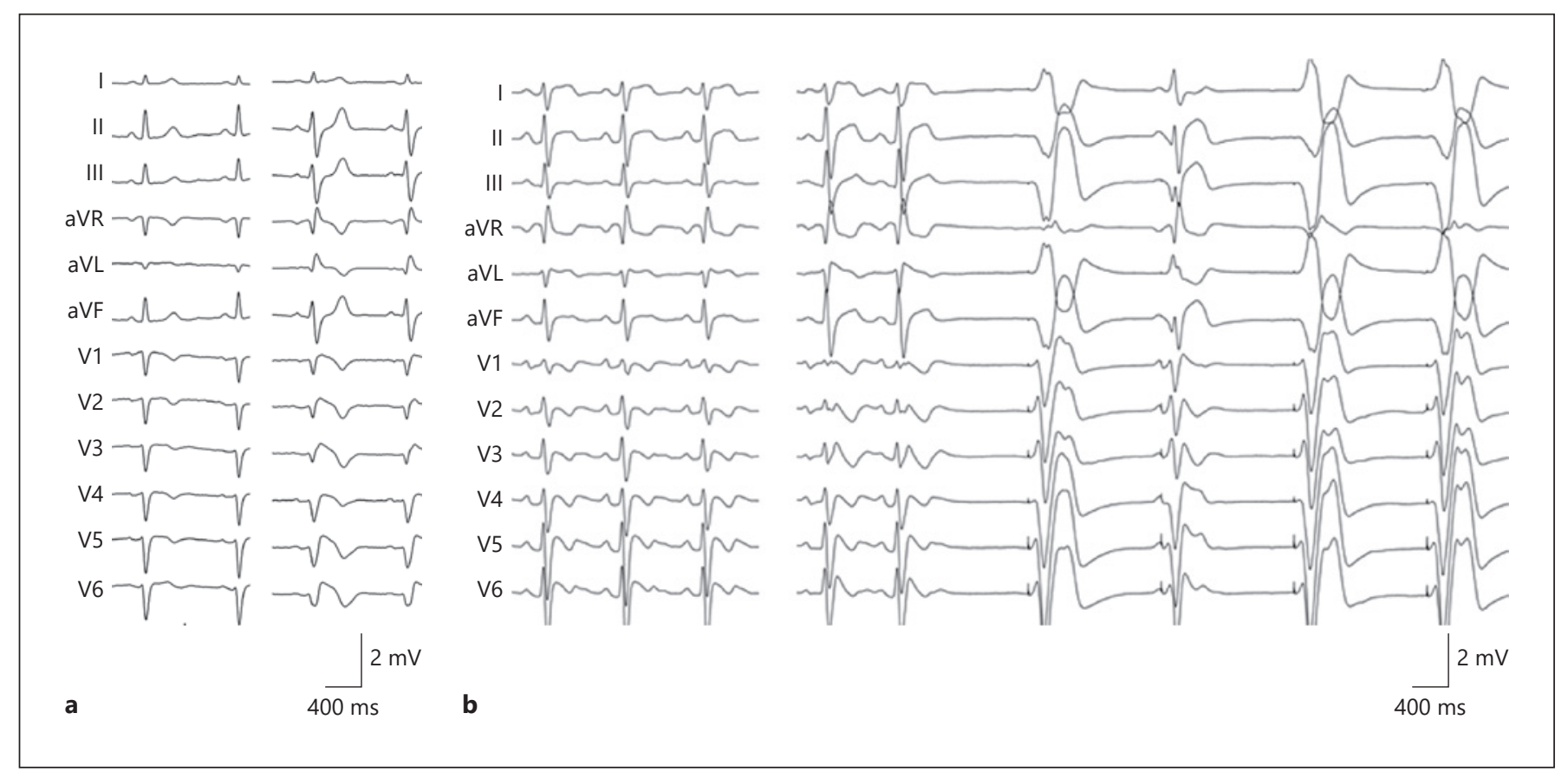

Fig. 2. a, b Ajmaline challenges in II-2 (a) and III-6 (b). Modified ECG with V1-6 precordial (V1-3 3rd ICS, V4-6 4th ICS) before (l) and after (r) administration of 30 and $35 \mathrm{mg}$ ajmaline, respectively.

\section{Clinical Reassessment}

At presentation in our outpatient clinic, we reviewed all clinical reports and ECGs available. While there were no pathological findings in the mother, several resting ECGs of her sons III-4 and III-6 were abnormal. In repeat ECGs, III-6 showed a sinus bradycardiac rhythm and prolonged PQ and QRS intervals for his age, with nonspecific depolarization and repolarization abnormalities (Fig. 1c). III-4 showed intermittent incomplete right bundle branch block with ST-elevations in V1 and V2 (Fig. 1d). Review of the emergency report of III-6 at the time of the syncope revealed the initial detected rhythm as bradycardia, with an escape rhythm of $40 / \mathrm{min}$. Intrahospital telemonitoring showed episodes of sinus bradycardia and a 2nd degree AV block (Fig. 1e). None of the reviewed ECGs showed a relevant prolongation or shortening of the QT interval. Due to the suspected familial channelopathy, we performed an ajmaline challenge in the mother. The test was positive with a Brugada type 1 ECG after administration of $35 \mathrm{mg}$ of ajmaline (Fig. 2a), allowing the diagnosis of familial BrS. III- 6 also produced a positive ajmaline $(30 \mathrm{mg})$ test performed at the age of 11 years, for example, ajmaline induced coved-type ECG elevations in the right precordial leads. At the same time, he developed sinus arrest with stimulation of the ICD/ pacemaker (Fig. 2b). Taken together, we found the clinical manifestation of ventricular fibrillation in III-4, SSS as well as cardiac conduction disease in III-6, sudden cardiac death in I-1, and an asymptomatic phenotype in the only female variant carrier II-2.

\section{In vitro Electrophysiology}

We performed whole-cell patch clamp recordings on HEK-293T cells transiently expressing wild-type Nav1.5 or Nav1.5-G1712S. We also examined Nav1.5-G1712C, as a previous study described this BrS-associated variant as a loss-of-function mutation [10]. Currents were evoked in cells held at $-120 \mathrm{mV}$ and challenged with $100-\mathrm{ms}$ long voltage steps from -120 to $+80 \mathrm{mV}$ at room temperature (Fig. 3b). The peak current amplitudes were reduced for Nav1.5-G1712S (3.1 $\pm 0.7 \mathrm{nA}, n=20)$ as compared to Nav1.5-WT $(8.3 \pm 1.4 \mathrm{nA}, n=19, p<0.01$, unpaired $t$-test, Fig. 3c). Nav1.5-G1712C indeed displayed a complete loss-of-function phenotype (Fig. 3b, c). Identical experiments at physiological temperatures (e.g., $35-37^{\circ} \mathrm{C}$ ) also gave smaller current amplitudes for Nav1.5-G1712S (3.7 $\pm 0.5 \mathrm{nA}, n=22)$ than for Nav1.5WT $(7.5 \pm 1.8 \mathrm{nA}, n=19, p<0.05$, unpaired $t$-test, Fig. $3 \mathrm{~d}$, e). However, the increase in temperature did not result in increased current amplitudes in any of the 2 constructs 


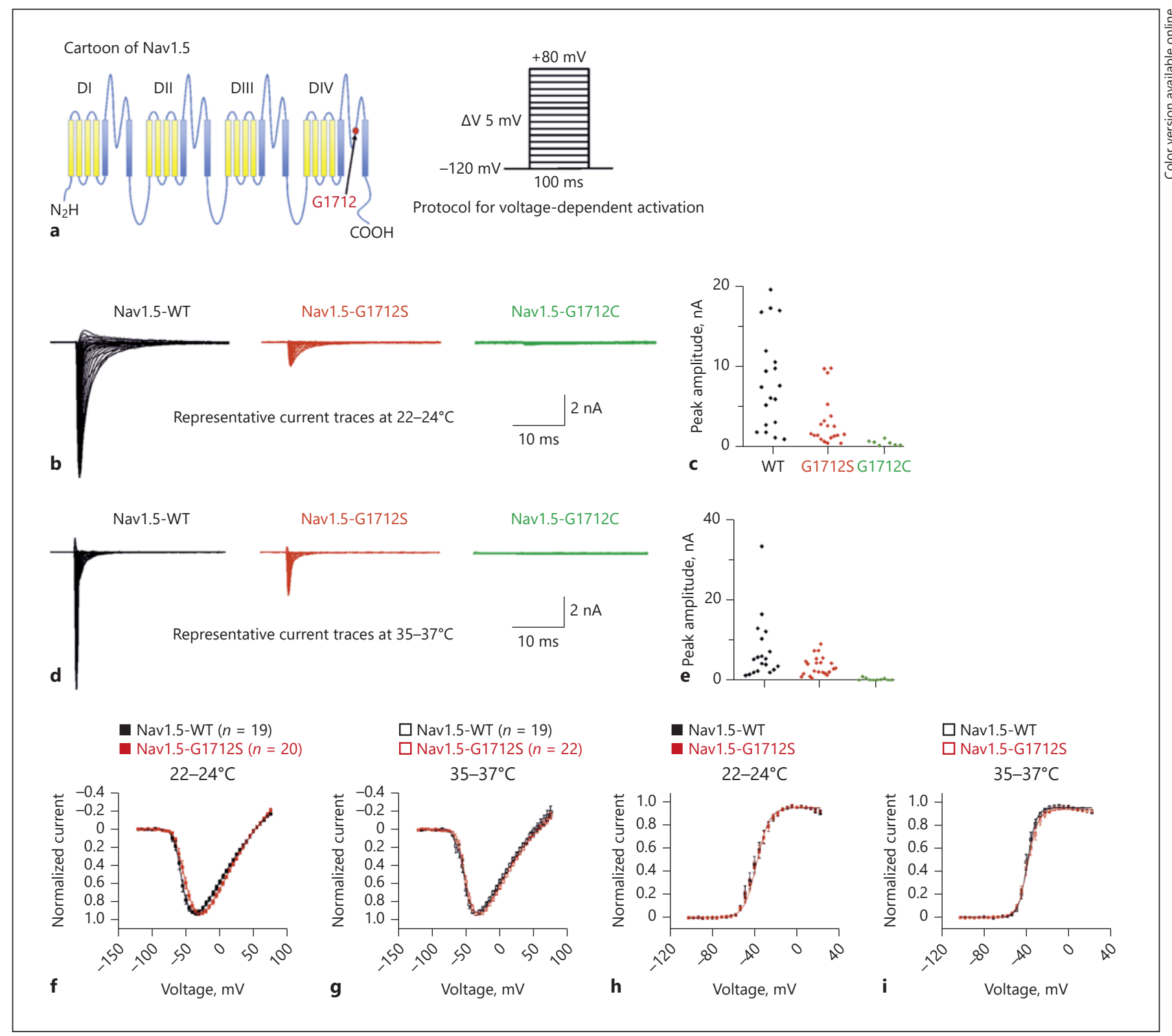

Fig. 3. a Cartoon of the $\alpha$-subunit Nav1.5 displaying the location of the residue G1712 in the pore-forming loop. b, d Representative current traces displaying voltage-dependent activation of Nav1.5WT (black, $n=19$ at both temperatures), Nav1.5-G1712S (red, $n=$ 20 respectively 22 ), and Nav1.5-G1712C (blue) at room (b, 22$\left.24^{\circ} \mathrm{C}\right)$ or near body $\left(\mathbf{d}, 35-37^{\circ} \mathrm{C}\right)$ temperature. Cells were held at $-120 \mathrm{mV}$ and 100-ms-long depolarizing pulses were applied, as de-

picted in the inset. c, e Peak current amplitudes of Nav1.5-WT-, Nav1.5-G1712S-, and Nav1.5-G1712C-mediated currents displayed as dot plots. Data are shown as mean \pm SEM. f, g Normalized current-voltage plots of Nav1.5-WT and Nav1.5-G1712S recorded at room temperature (f) or at body temperature $(\mathbf{g}) . \mathbf{h}, \mathbf{i}$ Conductancevoltage relationships converted from the peak IV-plots shown in figure in $\mathbf{f}$ and $\mathbf{g}$. Data were fitted with the Boltzmann function.

( $p=0.72$ for Nav1.5-WT and $p=0.45$ for Nav1.5-G1712S, unpaired $t$-tests). Plots of the current-voltage dependencies of Nav1.5-WT and Nav1.5-G1712S at both room and body temperature did not reveal any evident differences between Nav1.5-WT and Nav1.5-G1712S (Fig. 3f, g).

Moreover, no temperature-dependent effects on the voltage-dependent activation were observed (see online suppl.Fig. 1a, b; seewww.karger.com/doi/10.1159/000518210 for all online suppl. material). The conversion of these current-voltage plots into normalized conductance did 
Fig. 4. a, b Steady-state fast inactivation plots for Nav1.5-WT ( $n=18$, respectively, $19)$ and Nav1.5-G1712S ( $n=18$ at both temperatures) recorded at room (a, 22$24^{\circ}$ ) or near body (b, $\left.35-37^{\circ} \mathrm{C}\right)$ temperature. Cells were held at $-150 \mathrm{mV}$, and fast inactivation was induced by 100 -ms-long depolarizing pulses followed by the test pulse at $0 \mathrm{mV}$ (see inset). Mean data were normalized and fitted with the Boltzmann function. c, d Plots demonstrating recovery from fast inactivation of Nav1.5-WT $(n=$ 22 , respectively 25) and Nav1.5-G1712S ( $n$ $=18$, respectively, 17) recorded at room $(\mathbf{c}$, $\left.22-24^{\circ} \mathrm{C}\right)$ or near body $\left(\mathbf{d}, 35-37^{\circ} \mathrm{C}\right)$ temperature. Cells were held at $-150 \mathrm{mV}$ and two 20 -ms-long consecutive pulses to -10 $\mathrm{mV}$ with a variable interval ranging from 0.1 to $1,683 \mathrm{~ms}$ were applied (see inset). Normalized data were fitted with a single exponential to obtain the time constant $(\tau)$. e, f Plots displaying voltage-dependent slow inactivation for Nav1.5-WT ( $n=19$, respectively, 9) and Nav1.5-G1712S ( $n=$ 13 , respectively, 12) recorded at room (e, $\left.22-24^{\circ} \mathrm{C}\right)$ or near body $\left(\mathbf{f}, 35-37^{\circ} \mathrm{C}\right)$ temperature. Cells were held at $-150 \mathrm{mV}$, and slow inactivation was induced by 10 -s-long depolarizing pulses followed by an interpulse at $-150 \mathrm{mV}$ allowing recovery from fast inactivation and the test pulse at $0 \mathrm{mV}$ (see inset). Mean data were normalized, and a B-spline was drawn between data points to guide the eye. g, h. Plots demonstrating recovery from inactivation of Nav1.5-WT ( $n=10$, respectively, 11$)$ and Nav1.5-G1712S ( $n=11$, respectively, 12$)$ recorded at room $\left(\mathbf{g}, 22-24^{\circ} \mathrm{C}\right)$ or near body $\left(\mathbf{h}, 35-37^{\circ} \mathrm{C}\right)$ temperature. Cells were held at $-150 \mathrm{mV}$, and inactivation was induced by a 10 -s long pre-pulse at $0 \mathrm{mV}$, followed by recovery at $-150 \mathrm{mV}$ for a variable duration before the test pulse to $0 \mathrm{mV}$ was applied. Normalized data were fitted with a double exponential to obtain the time constants $\tau 1$ and $\tau 2$.

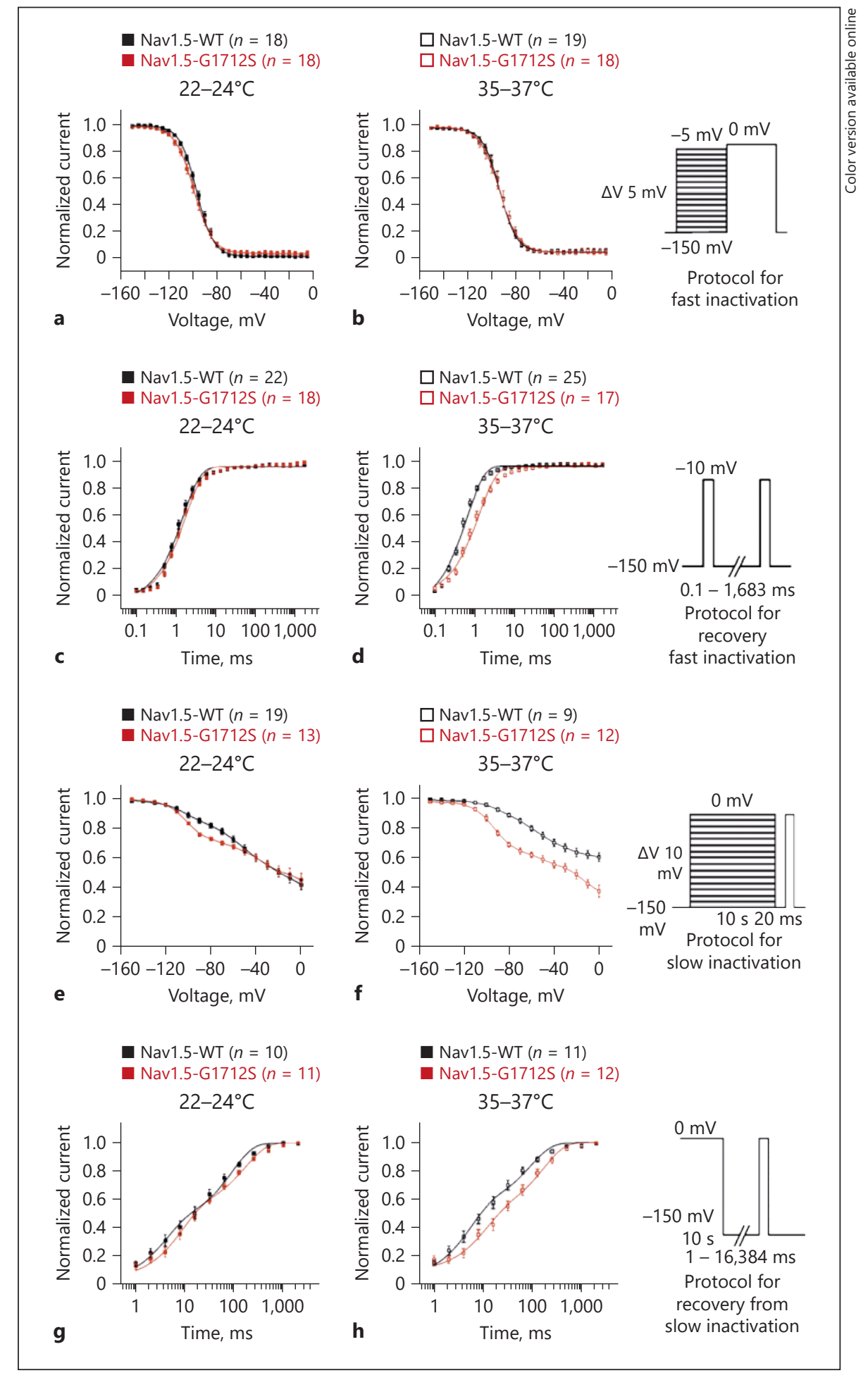

not reveal any differences between Nav1.5-WT $\left(22-24^{\circ} \mathrm{C}\right.$ : V0.5 $-47 \pm 0.9 \mathrm{mV} ; 35-37^{\circ} \mathrm{C}$ : $\left.\mathrm{V} 0.5-49 \pm 0.7 \mathrm{mV}\right)$ and Nav1.5-G1712S $\left(22-24^{\circ} \mathrm{C}\right.$ : V0.5 $-48 \pm 0.6 \mathrm{mV} ; 35-37^{\circ} \mathrm{C}$ : V0.5 $-47 \pm 0.8 \mathrm{mV}$, Fig. $3 \mathrm{~h}$, i) or temperature-dependent effects (online suppl. Fig. 1c, d). The voltage-dependent fast inactivation was explored by applying 100-ms-long depolarizing pre-pulses ranging from -150 to $-5 \mathrm{mV}$ in steps of $5 \mathrm{mV}$, followed by a test pulse to $0 \mathrm{mV}$. Nav1.5- 
WT and Nav1.5-G1712S displayed similar steady-state inactivation properties independent of temperature (room temperature WT: V0.5 $-96.7 \pm 0.3 \mathrm{mV}$, Hill coefficient $6.9 \pm 0.2, n=18$; G1712S: V0.5 $-99.4 \pm 0.2 \mathrm{mV}$, Hill coefficient $7.8 \pm 0.1, n=18$, Fig. 4a; body temperature: WT: V0.5 $-93.2 \pm 0.4 \mathrm{mV}$, Hill coefficient $6.9 \pm 0.2$, $n=19 ;$ G1712S: V0.5 $-93.7 \pm 0.5 \mathrm{mV}$, Hill coefficient 8.1 $\pm 0.3, n=18$, Fig. 4 b). In both genotypes, the increase in temperature resulted in a discrete shift of the steady-state inactivation curve toward more depolarized potentials (online suppl. Fig. 1e, f). We next assessed the recovery from fast inactivation by applying a protocol consisting of two 20 -ms-long consecutive pulses to $-10 \mathrm{mV}$ with a variable interval ranging from 0.1 to $1,683 \mathrm{~ms}$. At room temperature, the time dependencies of recovery from fast inactivation were not significantly different for Nav1.5WT ( $\tau: 1.5 \pm 0.1 \mathrm{~ms}, n=22)$ and Nav1.5-G1712S ( $\tau: 1.8 \pm$ $0.1 \mathrm{~ms}, n=18, p=0.052$ unpaired $t$-test, Fig. $4 \mathrm{c}$ ). At $35-$ $37^{\circ} \mathrm{C}$ however, the time course for recovery from fast inactivation was slower for Nav1.5-G1712S $(\tau: 1.3 \pm 0.08$ ms, $n=25)$ than Nav1.5-WT ( $\tau: 0.6 \pm 0.04 \mathrm{~ms}, n=17$, $p<0.05$ unpaired $t$-test, Fig. $4 \mathrm{~d})$. Thus, the increase in temperature revealed a robust phenotype of Nav1.5G1712S in case of recovery from inactivation. While both Nav1.5-WT ( $p<0.001$, unpaired $t$-test, online suppl. Fig. $1 \mathrm{~g})$ and Nav1.5-G1712S ( $p<0.01$, unpaired $t$-test, online suppl. Fig. 1h) recovered significantly faster upon heating, this effect seemed to be more robust on Nav1.5-WT than Nav1.5-G1712S. We next explored the voltage dependency of slow inactivation, which was induced by 10 $s$ long depolarizing pulses ranging from -150 to $0 \mathrm{mV}$. Before the test pulse to $0 \mathrm{mV}$ was applied, a 20-ms-long inter-pulse at $-150 \mathrm{mV}$ was inserted to allow recovery from fast inactivation. Both genotypes displayed incomplete slow inactivation with this protocol (Fig. 4e), making the calculation of the midpoint of slow-inactivation impossible. However, the fractions of inactivated channels after the final inactivating pulse to $0 \mathrm{mV}$ were similar for Nav1.5-WT ( $45 \pm 4 \%, n=19)$ and Nav1.5-G1712S (45 $\pm 5 \%, n=13, p=0.98$ unpaired $t$-test). At body temperature however, the remaining fraction of available channels was larger for Nav1.5-WT $(60 \pm 3 \%, n=9)$ than for Nav1.5-G1712S ( $42 \pm 4 \%, n=12, p<0.01$, unpaired $t$-test, Fig. 4f). Again, this emerging phenotype at body temperature was explained by the fact that Nav1.5-WT $(p<0.05$, unpaired $t$-test, online suppl. Fig. 1i) but not Nav1.5G1712S ( $p=0.56$, unpaired $t$-test, online suppl. Fig. $1 \mathrm{j}$ ) displayed less slow inactivation at $35-37^{\circ} \mathrm{C}$ than at $22-$ $24^{\circ} \mathrm{C}$. We finally applied a protocol examining recovery from both fast and slow inactivation. In cells held at -150
$\mathrm{mV}$, at 10-s-long pre-pulse was applied to fully inactivate the channels. Following recovery at $-150 \mathrm{mV}$ during a variable time (1-16,384 ms), available channels were assessed by a test pulse to $0 \mathrm{mV}$. Both genotypes displayed a biphasic recovery from inactivation (Fig. 3g, h). When best fitted with a double exponential function, the two time constants $\tau 1$ and $\tau 2$ were calculated to Nav1.5-WT: $\tau 1: 6.3 \pm 2.1 \mathrm{~ms}, \tau 2: 99.7 \pm 15.4 \mathrm{~ms}(n=10)$, and Nav1.5G1712S: $\tau 1: 8.3 \pm 1.9 \mathrm{~ms}, \tau 2: 155.6 \pm 19.7 \mathrm{~ms}(n=11)$ when explored at room temperature. While $\tau 1$ was not significantly different $(p=0.99), \tau 2$ was significantly slower for Nav1.5-G1712S than for Nav1.5-WT $(p<$ $0.019)$ (ANOVA one-way with Tukey post hoc test). At $35-37^{\circ} \mathrm{C}$, Nav1.5-G1712S ( $\tau 1: 11.2 \pm 2.7 \mathrm{~ms}, \tau 2: 141.0 \pm$ $16.6 \mathrm{~ms}, n=12$ ) exhibited a slower recovery than Nav1.5WT $(\tau 1: 3.9 \pm 0.6 \mathrm{~ms}, \tau 2: 77.2 \pm 12.2 \mathrm{~ms}, n=11)$. This difference was not significant for $\tau 1(p=0.96)$, but for $\tau 2$ $(p<0.001)$ (ANOVA one-way with Tukey post hoc test).

\section{Discussion}

We identified the missense mutation G1712S in SC$\mathrm{N} 5 \mathrm{~A}$ in a family with symptomatic $\mathrm{BrS}$ and found that the mutation causes a loss-of-function as Nav1.5-G1712S generated smaller inward currents than Nav1.5-WT. Surprisingly, an enhanced slow inactivation and an impeded recovery from fast and slow inactivation of Nav1.5G1712S became evident only when experiments were conducted at physiological temperatures. Thus, the mere analysis at room temperature may have promoted misinterpretation of Nav1.5-G1712S as a polymorphism with a more modest loss-of-function phenotype. In fact, similar biophysical properties have been proposed as pathogenic for other Brugada-causing Nav1.5 mutations $[2,9]$. Together with the clinical information and previously published in silico analysis, Nav1.5-G1712S can be declared as a pathogenic mutation with high probability to cause $\mathrm{BrS}$ as well as possibly SSS and cardiac conduction disease [5].

G1712S was previously reported to be associated with a Brugada phenotype in a single patient, and an in silico analysis had proposed a likely pathogenicity. However, that report lacked both clinical data and an electrophysiological characterization [6]. In fact, around $10 \%$ of published Brugada-associated SCN5A variants are suspected to be nonpathogenic [4]. The observation that some patients with a BrS phenotype completely lack Nav1.5-mutations has raised questions about the relevance of Nav1.5 mutations as causative for $\mathrm{BrS}$ [11]. This notion is sup- 
ported by studies revealing rather complex and in part Nav1.5-independent genetic backgrounds of BrS [12]. Nevertheless, our findings support the notion that any novel mutation detected warrants an extensive analysis, including electrophysiological experiments in order to define its possible pathogenicity [5]. Performing these electrophysiological experiments in transiently transfected HEK 293T cells however has several limitations. It may conceal properties of Nav1.5-G1712S expressed in native cardiomyocytes, known to express important interacting proteins, which could be relevant for BrS. Our analyses did not contain $\beta$-subunits, which modify the function of Nav1.5 [13]. The question if G1712S has an impact on the interaction with $\beta$-subunits warrants future exploration.

While a considerable number of Brugada-associated mutations have been identified within the pore loops and S5-S6 segments of Nav1.5, variants in these regions were rarely found in healthy controls [6]. By demonstrating the loss-of-function phenotypes of both Nav1.5-G1712S and G1712C located within the pore loop of domain IV, our data support this correlation between topology and pathogenicity. An important study from Li et al. [14] recently mapped known mutations of Nav1.5 associated with cardiac disorders based on a structure revealed by Cryo-EM. In fact, Nav1.5-G1712S was included in this study and was reported to be located in a segment which stabilizes the selectivity filter. A close-by mutation, Nav1.5-D1714G, was found to display similar effects on current density and slow inactivation in functional studies [9]. In regard to the temperature-sensitivity found in our study, Amin et al. [9] reported similar observations on Nav1.5-D1714G. Mutations in other regions have also shown temperature-dependent properties [8]. Previous studies have associated corresponding sites on other domains with altered slow inactivation properties [15]. Although the process of slow inactivation remains incompletely understood, a conformational change of the pore region is thought to be an underlying mechanism [16]. Voltage-dependent activation and steady-state fast inactivation were not affected in Nav1.5-G1712S. These channel properties have been associated with distinct regions on SCN5A [17]. We show that the G1712S exchange did influence recovery from fast inactivation. While this property has not been linked to the pore region, the mechanism is poorly understood and seems to be influenced by various locations.

Although a correlation between the genotype and phenotype in BrS does exist, evidence allowing geneticsbased risk stratification and decision-making is insufficient [18]. With 3 of 3 male family members being symp- tomatic with sudden cardiac arrest or syncope, our data support the notion that loss-of-function mutations on SCN5A located within the pore region are associated with a severe phenotype $[18,19]$. The occurrence of sinus arrest during ajmaline challenge further demonstrates the severity of the phenotype [20]. Ajmaline unmasking sinus node dysfunction has been described before [21]. Since overlap syndromes between BrS and SSS exist and may be caused by common SCN5A mutations, G1712S may cause BrS and SSS. This association has been described for only a few mutations, but none within the pore loop of domain IV so far. SSS tends to manifest earlier in life than the Brugada phenotype and shows male predominance. Some patients with SSS and BrS were even implanted a pacemaker, instead of an ICD [22-24]. Of note, the patient III- 6 not only showed features of SSS but also of cardiac conduction disease with prolonged PQ and QRS intervals at rest and documented intermittent 2nd degree AV block, further supporting the relevance of bradycardia in this case. Considering this and the ECG recordings of III-6, it is likely that the rhythmogenic syncope was caused by bradycardia, rather than tachycardia. Consequently, no further events occurred after implantation of the ICD/pacemaker. Van den Berg et al. [22] have previously reported several cases of bradycardia-associated death in patients with BrS. Together with our observation, these data suggest that clinicians should look out for clinically relevant bradycardia in patients with BrS. This is especially important when considering implantation of an ICD without anti-bradycardia function such as the subcutaneous ICD, which is increasingly considered in young patients with channelopathies [1].

\section{Conclusions}

Taken together, we characterized a new pathogenic SCN5A mutation, G1712S. It was associated with symptomatic BrS, cardiac conduction disease, and SSS requiring pacemaker therapy. The mutant caused a loss-offunction phenotype, but some effects were only obvious at physiological temperatures. Together with previous reports, our data suggest that reliable functional studies should be carried out at physiological temperature in addition to room temperature. Moreover, our findings stress the relevance of overlap syndromes and bradycardia in patients with BrS.
Nav1.5-G1712S Is a Variant of SCN5A

Associated with Brugada Syndrome
Cardiology 2021;146:754-762

DOI: $10.1159 / 000518210$ 


\section{Acknowledgements}

The authors thank Kerstin Reher, Marina Golombek, and Heike Bürger (Department of Anesthesiology) for excellent technical assistance.

\section{Statement of Ethics}

The study conforms to the Declaration of Helsinki. The study was approved by the Ethics Committee of Hannover Medical School (No. 1673-2013). The requirement of informed consent for this study was waived by the Institutional Committee on Human Research.

\section{Conflict of Interest Statement}

The authors have no conflicts of interest to declare.

\section{Funding Sources}

The study was supported by internal funds of Hannover Medical School.

\section{Author Contributions}

K.S. performed patch clamp recordings and wrote the manuscript. J.M.-L. analyzed clinical data and wrote the manuscript. C.Z. and D.V. analyzed clinical data. A.L. and C.V. planned und supervised the study, wrote, and corrected the manuscript.

\section{Data Availability Statement}

All data generated or analyzed during this study are included in this article and its online suppl. files. Further inquiries can be directed to the corresponding author.

\section{References}

1 Antzelevitch C, Yan GX, Ackerman MJ, Borggrefe M, Corrado D, Guo J, et al. J-Wave syndromes expert consensus conference report: emerging concepts and gaps in knowledge. J Arrhythm. 2016;32(5):315-39.

2 Veltmann C, Barajas-Martinez H, Wolpert C, Borggrefe M, Schimpf R, Pfeiffer R, et al. Further insights in the most common SCN5A mutation causing overlapping phenotype of long QT syndrome, Brugada syndrome, and conduction defect. J Am Heart Assoc. 2016;5:e003379.

3 Hosseini SM, Kim R, Udupa S, Costain G, Jobling R, Liston E, et al. Reappraisal of reported genes for sudden arrhythmic death: evidencebased evaluation of gene validity for Brugada syndrome. Circulation. 2018;138:1195-205.

4 Risgaard B, Jabbari R, Refsgaard L, Holst AG, Haunsø S, Sadjadieh A, et al. High prevalence of genetic variants previously associated with Brugada syndrome in new exome data. Clin Genet. 2013;84:489-95.

5 Campuzano O, Allegue C, Fernandez A, Iglesias A, Brugada R. Determining the pathogenicity of genetic variants associated with cardiac channelopathies. Sci Rep. 2015;5:7953.

6 Kapplinger JD, Tester DJ, Alders M, Benito B, Berthet $M$, Brugada J, et al. An international compendium of mutations in the SCN5A-encoded cardiac sodium channel in patients referred for Brugada syndrome genetic testing. Heart Rhythm. 2010;7:33-46.

7 Nagatomo T, Fan Z, Ye B, Tonkovich GS, January CT, Kyle JW, et al. Temperature dependence of early and late currents in human cardiac wildtype and long Q-T DeltaKPQ Na+ channels. Am J Physiol. 1998;275:H2016-24.

8 Dumaine R, Towbin JA, Brugada P, Vatta M, Nesterenko DV, Nesterenko VV, et al. Ionic mechanisms responsible for the electrocardiographic phenotype of the Brugada syndrome are temperature dependent. Circ Res. 1999;85:8039.
9 Amin AS, Verkerk AO, Bhuiyan ZA, Wilde AA, Tan HL. Novel Brugada syndrome-causing mutation in ion-conducting pore of cardiac $\mathrm{Na}+$ channel does not affect ion selectivity properties. Acta Physiol Scand. 2005;185:291-301.

10 Chen YY, Liu SR, Xie LZ, Zhu TY, Chen YZ Deng XJ, et al. [Functional analysis of a novel SCN5A mutation G1712C identified in Brugada syndrome]. Nan Fang Yi Ke Da Xue Xue Bao. 2016;37:256-60.

11 Probst V, Wilde AA, Barc J, Sacher F, Babuty D, Mabo P, et al. SCN5A mutations and the role of genetic background in the pathophysiology of Brugada syndrome. Circ Cardiovasc Genet. 2009;2:552-7.

12 Bezzina CR, Barc J, Mizusawa Y, Remme CA, Gourraud JB, Simonet F, et al. Common variants at SCN5A-SCN10A and HEY2 are associated with Brugada syndrome, a rare disease with high risk of sudden cardiac death. Nat Genet. 2013;45: 1044-9.

13 Savio-Galimberti E, Argenziano M, Antzelevitch C. Cardiac arrhythmias related to sodium channel dysfunction. Handb Exp Pharmacol. 2018;246:331-54.

14 Li Z, Jin X, Wu T, Zhao X, Wang W, Lie J, et al. Structure of human Na v 1.5 reveals the fast inactivation-related segments as a mutational hotspot for the long QT syndrome. Proc Natl Acad Sci USA. 2021;16(11):118.

15 Ong BH, Tomaselli GF, Balser JR. A structural rearrangement in the sodium channel pore linked to slow inactivation and use dependence. J Gen Physiol. 2000;116:653-62.

16 DeMarco KR, Clancy CE. Cardiac Na channels structure to function. Curr Top Membr. 2016; 78:287-311.

17 Patton DE, West JW, Catterall WA, Goldin AL. Amino acid residues required for fast $\mathrm{Na}(+)$ channel inactivation: charge neutralizations and deletions in the III-IV linker. Proc Natl Acad Sci U S A. 1992;89:10905-9.
18 Meregalli PG, Tan HL, Probst V, Koopmann TT, Tanck MW, Bhuiyan ZA, et al. Type of SCN5A mutation determines clinical severity and degree of conduction slowing in loss-of-function sodium channelopathies. Heart Rhythm. 2009;6: 341-8.

19 Moss AJ, Shimizu W, Wilde AA, Towbin JA, Zareba W, Robinson JL, et al. Clinical aspects of type-1 long-QT syndrome by location, coding type, and biophysical function of mutations involving the KCNQ1 gene. Circulation. 2007;115: 2481-9.

20 Morita H, Fukushima-Kusano K, Nagase S, Miyaji K, Hiramatsu S, Banba K, et al. Sinus node function in patients with Brugada-type ECG. Circ J. 2004;68:473-6.

21 Raviele A, Di Pede F, Zanocco A, Zuin G, Facin G, Mazzaro I, et al. [Predictive value of the ajmaline test in dysfunction of the sinus node. Prospective 4-year follow-up relative to 77 patients]. G Ital Cardiol. 1985;15:751-60.

22 van den Berg MP, Wilde AA, Viersma TJW, Brouwer J, Haaksma J, van der Hout AH, et al. Possible bradycardic mode of death and successful pacemaker treatment in a large family with features of long QT syndrome type 3 and Brugada syndrome. J Cardiovasc Electrophysiol. 2001;12:630-6.

23 Abe K, Machida T, Sumitomo N, Yamamoto H, Ohkubo K, Watanabe I, et al. Sodium channelopathy underlying familial sick sinus syndrome with early onset and predominantly male characteristics. Circ Arrhythm Electrophysiol. 2014; 7:511-7.

24 Makiyama T, Akao M, Tsuji K, Doi T, Ohno S, Takenaka K, et al. High risk for bradyarrhythmic complications in patients with Brugada syndrome caused by SCN5A gene mutations. J Am Coll Cardiol. 2005;46:2100-6. 